\title{
Clinical Effectiveness and Adverse Events of Bee Venom Therapy: A Systematic Review of Randomized Controlled Trials
}

\author{
Soobin Jang ${ }^{1}(\mathbb{D})$ and Kyeong Han Kim ${ }^{2, * \mathbb{D}}$ \\ 1 Clinical Medicine Division, Korea Institute of Oriental Medicine, Daejeon 34054 Korea; suebin@nate.com \\ 2 Department of Preventive Medicine, College of Korean Medicine, Woosuk University, Jeonju 54986, \\ Jeollabuk-do, Korea \\ * Correspondence: solip922@hanmail.net; Tel.: +82-63-290-9031; Fax: +82-63-291-1240
}

Received: 19 June 2020; Accepted: 27 August 2020; Published: 29 August 2020

\begin{abstract}
Bee venom has been used to treat many diseases because of its anti-inflammatory and analgesic effects. However, the secretions of bee venom can also cause life-threatening adverse reactions. The objective of this paper was to review the clinical effectiveness of bee venom and adverse events induced by bee venom, regardless of the disease. Four electronic databases were searched in April 2020. The reference lists of the retrieved articles and previous review articles were also hand-searched. Randomized controlled trials (RCTs) using any type of bee venom other than live bee stings for the clinical treatment of any disease other than cancer were included. The studies were selected, the data were extracted, and the quality of the studies was assessed by two authors. Risk of bias was assessed using the Cochrane risk of bias standards. Twelve RCTs were included in this review - three on Parkinson's disease, four on arthralgia, four on musculoskeletal disorders, and one on polycystic ovary syndrome. The types of bee venom used were acupuncture injections, ultrasound gel, and an ointment. Six studies reported adverse events, and skin reactions such as pruritus and swelling were the most common. The large-scale clinical trials of bee venom therapy are needed to verify the statistical difference, and the reporting system for adverse events is also required to increase the safety of bee venom therapy.
\end{abstract}

Keywords: bee venom; bee venom therapy; bee venom acupuncture; bee sting; efficacy; safety

Key Contribution: Bee venom has been used to treat diseases in the form of acupuncture, cream, and phonophoresis, and many related studies have been conducted. This study summarizes clinical effectiveness and adverse events of bee venom therapy through systematic review.

\section{Introduction}

Bee venom is secreted from worker bees and is one of the most well-known animal venoms. It consists of mast cell degranulation peptide, melittin, histamine, phospholipase A2 (PLA2), hyaluronidase, acid phosphatase, and nonpeptide components such as glucose and fructose [1]. In general, the $\mathrm{pH}$ of bee venom is 5.2 to 5.5 , which indicates acidity, and its specific gravity is 1.313 [2]. When bee venom enters the human body, various chemical agents of bee venom cause allergic reactions and, in severe cases, anaphylactic shock as a hypersensitivity $[3,4]$. PLA2 is the enzyme that is the major allergen in bee venom, and PLA2 secreted from bee venom is associated with inflammation and pain [5]. Bee venom derived PLA2.

Despite its toxicity, bee venom has also been utilized for therapeutic purposes in many clinical cases [6,7]. Bee venom therapy (BVT) is used for various diseases, and in particular, it is known to be effective for musculoskeletal diseases, including arthritis, arthralgia and immune-related diseases, 
because bee venom has anti-inflammatory and analgesic effects [8,9]. According to animal studies, bee venom has pharmacological effects, including the inhibition of cyclo-oxygenase-2, expression of PLA2 and reduction of tumor necrosis factor $\alpha$, interleukin (IL)-1, IL-6, nitric oxide, and oxygen-reactive species $[10,11]$.

There are several types of BVT-live bee stings, bee venom acupuncture (BVA) or injections, and externally applied bee venom ointments. Live bee stings, a traditional method of BVT, are applied directly on human skin; therefore, they have a high risk of adverse reactions. BVA is applied by injecting bee venom diluted to a ratio of less than 1:10,000 to minimize the side effects while providing therapeutic effects [2]. A major difference between BVA and bee venom injections is that BVA is administered on the basis of acupoints.

According to a previous review of adverse reactions from bee venom [12], 184 adverse events (AEs) were reported among 397 patients who were exposed to bee venom in 20 randomized controlled trials. Bee venom should be carefully evaluated as a medical treatment because it can cause harm as well as medical benefits. Therefore, this systematic review aimed to summarize the clinical effectiveness of bee venom and AEs induced by bee venom, regardless of the disease.

\section{Methods}

\subsection{Study Registration}

The protocol of this review was registered in PROSPERO (registration number CRD42020165821).

\subsection{Search Method for Identifying the Studies}

This study included the following 4 electronic databases-Medline via Pubmed, Embase via Elsevier, the Cochrane Library, and Web of Science. The reference lists of the retrieved articles were hand-searched, and previous review articles were also examined.

The key words used for the search were 'bee venom' and 'bee venom acupuncture'. Only articles written in English and published since 2010 were included. The search was conducted on 6 April 2020.

\subsection{Inclusion Criteria for This Review}

\subsubsection{Types of Studies}

This systematic review included only randomized controlled trials (RCTs) that were peer-reviewed. Studies with other designs, such as observational studies, cohort studies, case reports, case series, non-RCTs, animal and experimental studies, and theses were excluded.

\subsubsection{Types of Participants}

Regarding the participants, only those with cancer were excluded. Since chemotherapy can cause several side effects in cancer patients, they were not included in the review of the adverse events of bee venom.

\subsubsection{Types of Interventions}

All types of bee venom treatments (i.e., bee venom acupuncture, bee venom injections, bee venom cream) were included in this review. Live bee stings and propolis were excluded. RCTs that combined a bee venom intervention with other interventions were also excluded. RCTs in which other interventions (e.g., drugs, massage, and exercise) were administered to all groups in the exact same manner were included.

There were no restrictions on the comparisons. Placebos, active-control groups, no-treatment groups, and wait-list control groups were allowed as control groups. 


\subsubsection{Type of Outcome Measures}

All outcomes reflecting the clinical effectiveness of bee venom were included. However, RCTs that assessed only the treatment rate without any clinical outcomes were excluded from this review. Articles that did not report the measured values before and after treatment were also excluded.

\subsection{Data Collection, Extraction and Assessment}

\subsubsection{Selection of Studies}

Two authors (SJ and KHK) independently screened the titles and abstracts of the studies retrieved from 4 databases after excluding the duplicate articles. Then, the full texts of the selected articles were reviewed to ensure that each article met the inclusion criteria for this review. When two authors had a difference of opinion, a third reviewer resolved the disagreement. The entire process is summarized in a flow diagram based on the Preferred Reporting Items for Systematic Reviews and Meta-Analyses (PRISMA) guidelines (Figure 1) [13].
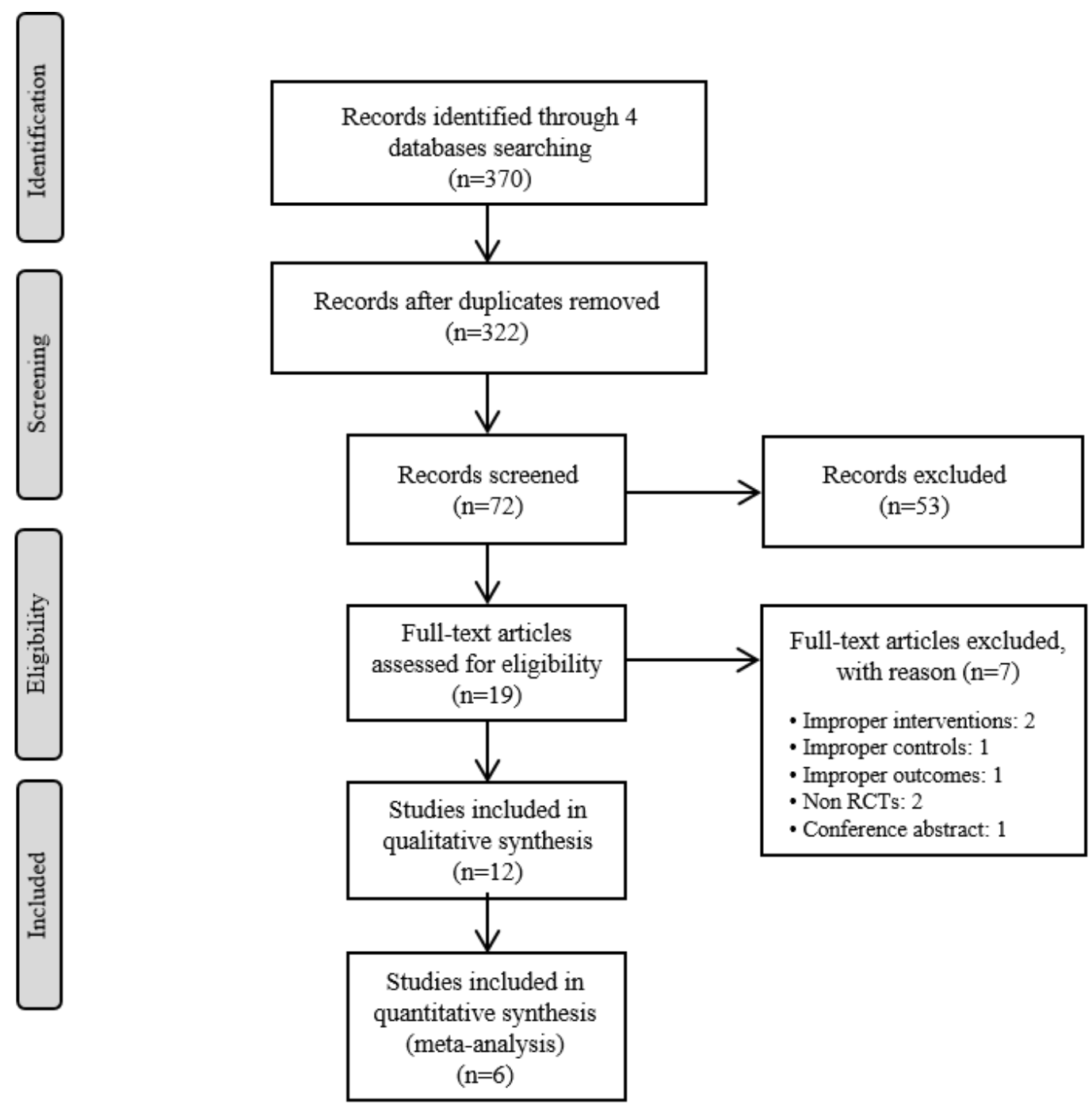

Figure 1. The PRISMA Flow Diagram of Study Selection. 


\subsubsection{Data Extraction}

One author (SJ) extracted the data, and another author (KHK) reviewed the extracted data. Basic information such as the participants, interventions, comparisons, outcomes and adverse events for each included study were recorded.

\subsubsection{Assessment of Risk of Bias}

Two reviewers (SJ and KHK) assessed the quality of the included studies using the risk of bias (RoB) tool that was developed by Cochrane [14]. RoB was assessed on the basis of the following seven items-(1) random sequence generation; (2) allocation concealment; (3) blinding of participants and personnel; (4) blinding of outcome assessment; (5) completeness of outcome data; (6) completeness of reporting; and (7) other sources of bias. Each item of every RCT was categorized as "high risk $(\mathrm{H})$ ", "unclear (U)", or "low risk (L)" for all studies. An RoB graph was generated by Review Manager (Cochrane Collaboration Software, RevMan), version 5.3.

\section{Results}

\subsection{Description of Included Trials}

From the four databases that were searched, 370 records were identified, and 322 articles remained after duplicates were removed. After the titles and abstracts were screened, 72 articles remained; after the full-texts were reviewed, 12 RCTs [15-26] were included in this review. A flow chart of the study selection process, including the reasons for exclusion, is shown in Figure 1. Among the 12 RCTs, eight were conducted in South Korea [15,17-19,21-23,25], two in Egypt [20,26], one in France [16], and one in Poland [24]. The number of participants ranged from 20 to 367. Four studies [15,17-19] were three-arm clinical trials, and one study [19] included 1-year follow-up results for another study [18]. The treated conditions were Parkinson's disease, low back pain, temporomandibular disorder (RDC/TMD Ia and RDC/TMD Ib), delayed onset muscle soreness, adhesive capsulitis, pelvic inflammatory disease, knee osteoarthritis, and polycystic ovary syndrome.

The types of bee venom treatments used in the RCTs were acupuncture, ointment, and ultrasound gel. BVA, which is the most typical type of BVT, not only involves stimulation, as does acupuncture, but also involves the injection of bee venom fluids. The bee venom fluid was prepared by dilution in distilled water or $\mathrm{NaCl}$ (normal saline). Ointments and ultrasound gel, which are externally applied, were used for the treatment of muscle soreness and temporomandibular disorder.

Placebo, active-control, and no-treatment control groups were included. Normal saline instead of bee venom was injected in the placebo group in six trials [16-19,22,23], and histamine phosphate was used in one trial [21]. Conventional drugs, acupuncture, Vaseline, and ultrasound without bee venom gel were used in the control groups. The details are described in Table 1. 
Table 1. Basic characteristics of included studies

\begin{tabular}{|c|c|c|c|c|c|c|c|c|c|c|}
\hline Condition & $\begin{array}{l}\text { Author } \\
\text { [Ref] Year }\end{array}$ & $\begin{array}{l}\text { Conducting } \\
\text { Country }\end{array}$ & $\begin{array}{l}\text { Age } \\
\text { [Mean] }\end{array}$ & $\begin{array}{l}\text { Sex } \\
{[M / F]}\end{array}$ & $\begin{array}{l}\text { Study } \\
\text { Period }\end{array}$ & $\begin{array}{l}\text { Type of Bee } \\
\text { Venom }\end{array}$ & Intervention & Control & Outcome & $\begin{array}{l}\text { Results } \\
\text { ( } p \text { Value } \\
\text { between Two } \\
\text { Groups) }\end{array}$ \\
\hline \multirow{3}{*}{$\begin{array}{l}\text { Parkinson's } \\
\text { disease }\end{array}$} & $\begin{array}{l}\text { Cho et al. } \\
\text { [15] } 2012\end{array}$ & $\begin{array}{l}\text { South } \\
\text { Korea }\end{array}$ & $\begin{array}{l}\text { (A) } 57.0 \\
\text { (B) } 55.0 \\
\text { (C) } 57.0\end{array}$ & $\begin{array}{l}\text { (A) } 5 / 8 \\
\text { (B) } 5 / 8 \\
\text { (C) } 3 / 6\end{array}$ & 8 weeks & $\begin{array}{l}\text { Acupuncture } \\
\text { (Injection) }\end{array}$ & $\begin{array}{l}\text { (A) } \\
\text { - BVA at bilateral GB20, } \\
\text { LI11, GB 34, ST36, } \\
\text { and LR3, } \\
\text { - BV } 0.1 \text { mL diluted on } \\
\text { 1:20,000 distilled water } \\
\text { - twice a week }\end{array}$ & $\begin{array}{l}\text { (B) } \\
\text { - Acupuncture at } \\
\text { same points with } \\
\text { group A } \\
\text { - twice a week } \\
\text { (C) } \\
\text { No treatment }\end{array}$ & $\begin{array}{l}\text { (1) UPDRS } \\
\text { (2) PDQL } \\
\text { (3) BDI } \\
\text { (4) BBS } \\
\text { (5) time of steps } \\
\text { required to walk } 30 \mathrm{~m} \\
\text { (6) number of steps } \\
\text { required to walk } 30 \mathrm{~m}\end{array}$ & $\begin{array}{l}\text { [A-B, A-C] } \\
\text { (1) NS, } p<0.05 \\
\text { (2) NS, NS } \\
\text { (3) NS, NS } \\
\text { (4) NS, NS } \\
\text { (5) NS, NS } \\
\text { (6) NS, NS }\end{array}$ \\
\hline & $\begin{array}{l}\text { Hartmann } \\
\text { et al. [16] } \\
2016\end{array}$ & France & $\begin{array}{l}\text { (A) } 60.3 \\
\text { (median) } \\
\text { (B) } 63.3 \\
\text { (median) }\end{array}$ & $\begin{array}{l}\text { (A) } 8 / 12 \\
\text { (B) } 12 / 8\end{array}$ & $\begin{array}{l}11 \\
\text { months }\end{array}$ & Injection & $\begin{array}{l}\text { (A) } \\
-\mathrm{BV} 0.1 \mathrm{mg} \text { diluted in } 1 \\
\mathrm{~mL} \text { of } \mathrm{NaCl} 0.9 \% \\
\text { - once a month }\end{array}$ & $\begin{array}{l}\text { (B) } \\
\text { - Placebo (normal } \\
\text { saline } 1 \mathrm{~mL}) \\
\text { - once a month }\end{array}$ & $\begin{array}{l}\text { (1) UPDRS } \\
\text { (2) Hoenh and Yahr } \\
\text { stages } \\
\text { (3) ADL } \\
\text { (4) BREF } \\
\text { (5) MMS } \\
\text { (6) PDQ-39 } \\
\text { (7) [123I]-FP-CIT } \\
\text { binding potential }\end{array}$ & $\begin{array}{l}\text { (1) NS } \\
\text { (2) NS } \\
\text { (3) NS } \\
\text { (4) NS } \\
\text { (5) NS } \\
\text { (6) NS } \\
\text { (7) NS }\end{array}$ \\
\hline & $\begin{array}{l}\text { Cho et al. } \\
\text { [17] } 2018\end{array}$ & $\begin{array}{l}\text { South } \\
\text { Korea }\end{array}$ & $\begin{array}{l}\text { (A) } 64.4 \\
\text { (B) } 61.3 \\
\text { (C) } 64.1\end{array}$ & $\begin{array}{l}\text { (A) } 14 / 10 \\
\text { (B) } 8 / 16 \\
\text { (C) } 10 / 5\end{array}$ & $\begin{array}{l}12 \text { weeks } \\
+8 \text { weeks } \\
\text { (follow-up) }\end{array}$ & $\begin{array}{l}\text { Acupuncture } \\
\text { (Injection) }\end{array}$ & $\begin{array}{l}\text { (A) } \\
\text { - BVA at bilateral GB20, } \\
\text { LI11, GB 34, ST36, } \\
\text { and LR3 } \\
\text { - dried BV } 1 \mathrm{mg} \text { diluted } \\
\text { in } 20 \mathrm{~mL} \text { of normal } \\
\text { saline } \\
\text { - twice a week }\end{array}$ & $\begin{array}{l}\text { (B) } \\
\text { - Placebo (normal } \\
\text { saline injection at } \\
\text { same points with } \\
\text { group A } \\
\text { - twice a week } \\
\text { (C) } \\
\text { Antiparkinsonian } \\
\text { drugs }\end{array}$ & $\begin{array}{l}\text { (1) UPDRS } \\
\text { (2) PDQL } \\
\text { (3) BDI } \\
\text { (4) PIGD } \\
\text { (5) Gait speed } \\
\text { (6) Gait number } \\
\text { (7) MXE } \\
\text { (8) DCL }\end{array}$ & $\begin{array}{l}\text { [A-B, A-C] } \\
\text { (1) NS, } p=0.001 \\
\text { (2) NS, } p=0.968 \\
\text { (3) NS, NS } \\
\text { (4) NS, } p=0.001 \\
\text { (5) NS, NS } \\
\text { (6) NS, } p=0.115 \\
\text { (7) NS, NS } \\
\text { (8) NS, NS }\end{array}$ \\
\hline
\end{tabular}


Table 1. Cont.

\begin{tabular}{|c|c|c|c|c|c|c|c|c|c|c|}
\hline Condition & $\begin{array}{l}\text { Author } \\
\text { [Ref] Year }\end{array}$ & $\begin{array}{l}\text { Conducting } \\
\text { Country }\end{array}$ & $\begin{array}{l}\text { Age } \\
\text { [Mean] }\end{array}$ & $\begin{array}{l}\text { Sex } \\
{[M / F]}\end{array}$ & $\begin{array}{l}\text { Study } \\
\text { Period }\end{array}$ & $\begin{array}{l}\text { Type of Bee } \\
\text { Venom }\end{array}$ & Intervention & Control & Outcome & $\begin{array}{l}\text { Results } \\
\text { ( } p \text { Value } \\
\text { between Two } \\
\text { Groups) }\end{array}$ \\
\hline \multirow[t]{2}{*}{$\begin{array}{l}\text { Adhesive } \\
\text { capsulitis }\end{array}$} & $\begin{array}{l}\text { Koh et al. } \\
\text { [18] } 2013\end{array}$ & \multirow[t]{2}{*}{$\begin{array}{l}\text { South } \\
\text { Korea }\end{array}$} & $\begin{array}{l}\text { (A) } 55.0 \\
\text { (B) } 56.1 \\
\text { (C) } 55.1\end{array}$ & $\begin{array}{l}\text { (A) } 6 / 16 \\
\text { (B) } 8 / 15 \\
\text { (C) } 6 / 17\end{array}$ & 12 weeks & \multirow[t]{2}{*}{$\begin{array}{l}\text { Acupuncture } \\
\text { (Injection) }\end{array}$} & \multirow{2}{*}{$\begin{array}{l}\text { (A) } \\
\text { - BVA } 100 \text { cc (1:10,000 of } \\
\text { saline) at LI15, LI16, } \\
\text { TE14, GB21, C7, SI11, } \\
\text { and additional 5 points } \\
\text { around the shoulder } \\
\text { - Physiotherapy } \\
\text { (B) } \\
\text { - BVA } 300 \text { cc (1:30,000 of } \\
\text { saline) at same points } \\
\text { with group A } \\
\text { - Physiotherapy }\end{array}$} & \multirow[t]{2}{*}{$\begin{array}{l}\text { (C) } \\
\text { - Placebo (normal } \\
\text { saline injection) } \\
\text { - Physiotherapy }\end{array}$} & $\begin{array}{l}\text { (1) SPADI } \\
\text { (2) VAS-at rest, } \\
\text { at night, motion } \\
\text { (3) ROM-abduction, } \\
\text { forward flexion, } \\
\text { extension, } \\
\text { external rotation }\end{array}$ & $\begin{array}{l}\text { [Among } 3 \\
\text { groups] } \\
\text { (1) } p=0.017 \\
\text { (2) } p=0.110 \\
\text { (at rest), } \\
p=0.160 \\
\text { (at night), } \\
p=0.029 \\
\text { (motion) } \\
\text { (3) NS }\end{array}$ \\
\hline & $\begin{array}{l}\text { Park et al. } \\
\text { [19] 2014, } \\
\text { follow-up of } \\
\text { [18] }\end{array}$ & & $\begin{array}{l}\text { (A) } 55.4 \\
\text { (B) } 52.8 \\
\text { (C) } 56.4\end{array}$ & $\begin{array}{l}\text { (A) } 6 / 16 \\
\text { (B) } 8 / 15 \\
\text { (C) } 6 / 17\end{array}$ & 1 year & & & & $\begin{array}{l}\text { 91) SPADI } \\
\text { (2) VAS-at rest, } \\
\text { at night, motion } \\
\text { (3) Recurrence of } \\
\text { symptoms }\end{array}$ & $\begin{array}{l}\text { [Among } 3 \\
\text { groups] } \\
\text { (1) } p=0.043 \\
\text { (2) NS } \\
\text { (3) A-1, B-3, C-4 }\end{array}$ \\
\hline $\begin{array}{l}\text { Pelvic } \\
\text { inflammatory } \\
\text { disease }\end{array}$ & $\begin{array}{l}\text { Mohamed } \\
\text { et al. [20] } \\
2016\end{array}$ & Egypt & $\begin{array}{l}\text { (A) } 32.1 \\
\text { (B) } 32.5\end{array}$ & $\begin{array}{l}\text { (A) } 0 / 15 \\
\text { (B) } 0 / 15\end{array}$ & 4 weeks & $\begin{array}{l}\text { Ultrasound } \\
\text { gel }\end{array}$ & $\begin{array}{l}\text { (A) } \\
\text { - BV gel topical } \\
\text { application with } \\
\text { phonophoresis } \\
\text { - BV } 20 \mu \mathrm{g} / \mathrm{gel} 1 \mathrm{~g} \\
-20 \mathrm{~min} / \mathrm{time}, 3 \text { times } \\
\text { per week } \\
\text { - doxycycline } 100 \mathrm{mg} \text {, } \\
\text { orally bid for } 7 \text { days }\end{array}$ & $\begin{array}{l}\text { (B) } \\
\text { Doxycycline } \\
100 \text { mg, orally bid } \\
\text { for } 7 \text { days }\end{array}$ & $\begin{array}{l}\text { (1) C-reactive protein } \\
\text { (2) Pain intensity }\end{array}$ & $\begin{array}{l}\text { (1) } p<0.0001 \\
\text { (2) } p<0.0001\end{array}$ \\
\hline
\end{tabular}


Table 1. Cont

\begin{tabular}{|c|c|c|c|c|c|c|c|c|c|c|}
\hline Condition & $\begin{array}{l}\text { Author } \\
\text { [Ref] Year }\end{array}$ & $\begin{array}{l}\text { Conducting } \\
\text { Country }\end{array}$ & $\begin{array}{l}\text { Age } \\
\text { [Mean] }\end{array}$ & $\begin{array}{l}\text { Sex } \\
{[M / F]}\end{array}$ & $\begin{array}{l}\text { Study } \\
\text { Period }\end{array}$ & $\begin{array}{l}\text { Type of Bee } \\
\text { Venom }\end{array}$ & Intervention & Control & Outcome & $\begin{array}{l}\text { Results } \\
\text { ( } p \text { Value } \\
\text { between Two } \\
\text { Groups) }\end{array}$ \\
\hline $\begin{array}{l}\text { Knee } \\
\text { osteoarthritis }\end{array}$ & $\begin{array}{l}\text { Conrad et al. } \\
\text { [21] } 2019\end{array}$ & $\begin{array}{l}\text { South } \\
\text { Korea }\end{array}$ & $\begin{array}{l}\text { (A) } 56.9 \\
\text { (B) } 55.8\end{array}$ & $\begin{array}{l}\text { (A) } \\
91 / 143 \\
\text { (B) } 64 / 69\end{array}$ & 12 weeks & Injection & $\begin{array}{l}\text { (A) } \\
\text { - BV injection at } 2-15 \\
\text { sites escalating over } \\
\text { study period ( } 0.1 \mathrm{~mL} \text { at } \\
\text { each site), } 10 \text { points at } \\
\text { bilateral knees, BL19, } \\
\text { BL21, BL23, BL25, } \\
\text { and BL27 } \\
\text { - dried HV } 1.0 \mathrm{mg} / \\
1.0 \mathrm{~mL} 0.5 \% \\
\text { preservative-free } \\
\text { lidocaine }\end{array}$ & $\begin{array}{l}\text { (B) } \\
\text { Histamine } \\
\text { phosphate } \\
\text { injection at same } \\
\text { sites with group A }\end{array}$ & $\begin{array}{l}\text { (1-1) WOMAC pain } \\
\text { (1-2) WOMAC } \\
\text { physical function } \\
\text { (2-1) WOMAC VAS } \\
\text { resting } \\
\text { (2-2) WOMAC VAS } \\
\text { walking } \\
\text { (3) PGA }\end{array}$ & $\begin{array}{l}\text { (1-1) } p=0.001 \\
(1-2) p=0.001 \\
(2-1) p=0.1051 \\
\text { (2-2) } p=0.001 \\
\text { (3) } p=0.0001\end{array}$ \\
\hline \multirow[b]{2}{*}{$\begin{array}{l}\text { Low back } \\
\text { pain }\end{array}$} & $\begin{array}{l}\text { Shin et al. } \\
\text { [22] } 2012\end{array}$ & $\begin{array}{l}\text { South } \\
\text { Korea }\end{array}$ & $\begin{array}{l}\text { (A) } 42.9 \\
\text { (B) } 40.0\end{array}$ & $\begin{array}{l}\text { (A) } 13 / 17 \\
\text { (B) } 14 / 16\end{array}$ & 4 weeks & $\begin{array}{l}\text { Acupuncture } \\
\text { (Injection) }\end{array}$ & $\begin{array}{l}\text { (A) } \\
\text { - BVA at bilateral BL23, } \\
\text { BL24, and BL25 } \\
\text { - BV 0.1 mL diluted on } \\
\text { 1:2000 distilled water } \\
\text { - twice a week }\end{array}$ & $\begin{array}{l}\text { (B) } \\
\text { - Placebo (normal } \\
\text { saline injection at } \\
\text { same points with } \\
\text { group A) } \\
\text { - twice a week }\end{array}$ & $\begin{array}{l}\text { (1) Pain intensity } \\
\text { (VAS) } \\
\text { (2) ODQ } \\
\text { (3) SF-36 }\end{array}$ & $\begin{array}{l}\text { (1) } p=0.012 \\
\text { (2) NS } \\
\text { (3) NS }\end{array}$ \\
\hline & $\begin{array}{l}\text { Seo et al. } \\
\text { [23] } 2017\end{array}$ & $\begin{array}{l}\text { South } \\
\text { Korea }\end{array}$ & $\begin{array}{l}\text { (A) } 49.9 \\
\text { (B) } 50.1\end{array}$ & $\begin{array}{l}\text { (A) } 9 / 18 \\
\text { (B) } 4 / 23\end{array}$ & 3 weeks & $\begin{array}{l}\text { Acupuncture } \\
\text { (Injection) }\end{array}$ & $\begin{array}{l}\text { (A) } \\
\text { - BVA at bilateral BL23, } \\
\text { BL24, BL26, and GB30, } \\
\text { GV3, GV4, and GV5 } \\
\text { - dried BV diluted on } \\
\text { 1:20000 normal saline } \\
(0.9 \% \mathrm{NaCl}) \\
\text { - Loxonin } 60 \mathrm{mg} \text {, } \\
\text { orally tid for } 3 \text { weeks }\end{array}$ & $\begin{array}{l}\text { (B) } \\
\text { - Placebo (normal } \\
\text { saline injection at } \\
\text { same points with } \\
\text { group A) } \\
\text { - Loxonin } 60 \mathrm{mg} \text {, } \\
\text { orally tid for } \\
3 \text { weeks }\end{array}$ & $\begin{array}{l}\text { (1) Bothersomeness } \\
\text { (VAS) } \\
\text { (2) Pain intensity } \\
\text { (VAS) } \\
\text { (3) ODI } \\
\text { (4) BDI } \\
\text { (5) EQ-5D }\end{array}$ & $\begin{array}{l}\text { (1) } p=0.016 \\
\text { (2) } p=0.049 \\
\text { (3) } p=0.009 \\
\text { (4) } p=0.043 \\
\text { (5) } p=0.051\end{array}$ \\
\hline
\end{tabular}


Table 1. Cont.

\begin{tabular}{|c|c|c|c|c|c|c|c|c|c|c|}
\hline Condition & $\begin{array}{l}\text { Author } \\
\text { [Ref] Year }\end{array}$ & $\begin{array}{l}\text { Conducting } \\
\text { Country }\end{array}$ & $\begin{array}{l}\text { Age } \\
\text { [Mean] }\end{array}$ & $\begin{array}{l}\text { Sex } \\
{[M / F]}\end{array}$ & $\begin{array}{l}\text { Study } \\
\text { Period }\end{array}$ & $\begin{array}{l}\text { Type of Bee } \\
\text { Venom }\end{array}$ & Intervention & Control & Outcome & $\begin{array}{l}\text { Results } \\
\text { ( } p \text { Value } \\
\text { between Two } \\
\text { Groups) }\end{array}$ \\
\hline $\begin{array}{l}\text { Temporoma } \\
\text { ndibular } \\
\text { disorder } \\
\text { (RDC/TMD } \\
\text { Ia and } \\
\text { RDC/TMD } \\
\text { Ib) }\end{array}$ & $\begin{array}{l}\text { Nitecka } \\
\text {-Buchta et al. } \\
\text { [24] } 2014\end{array}$ & Poland & $\begin{array}{l}23 \\
(22-34)\end{array}$ & $\begin{array}{l}\text { (A) } 6 / 28 \\
\text { (B) } 4 / 30\end{array}$ & 2 weeks & Ointment & $\begin{array}{l}\text { (A) } \\
\text { - BV ointment ( } 0.012 \mathrm{mg} \\
\text { liquid BV) for topical } \\
\text { skin application in } \\
\text { region of both masseter } \\
\text { muscles } \\
\text { - Massage }\end{array}$ & $\begin{array}{l}\text { (B) } \\
\text { - Vaseline at same } \\
\text { region with } \\
\text { group A } \\
\text { - Massage }\end{array}$ & $\begin{array}{l}\text { (1) Muscle tonus } \\
\text { (2) Muscle contraction } \\
\text { (3) VAS }\end{array}$ & $\begin{array}{l}\text { (1) NR } \\
\text { (2) NR } \\
\text { (3) NR }\end{array}$ \\
\hline $\begin{array}{l}\text { Delayed } \\
\text { onset muscle } \\
\text { soreness }\end{array}$ & $\begin{array}{l}\text { Kim et al. } \\
\text { [25] } 2014\end{array}$ & $\begin{array}{l}\text { South } \\
\text { Korea }\end{array}$ & $\begin{array}{l}\text { (A) } 27.4 \\
\text { (B) } 28.9\end{array}$ & $\begin{array}{l}\text { (A) } 0 / 10 \\
\text { (B) } 0 / 10\end{array}$ & 3 days & $\begin{array}{l}\text { Ultrasound } \\
\text { gel }\end{array}$ & $\begin{array}{l}\text { (A) } \\
\text { - Ultrasound gel and } \\
\text { diluted bee venom } \\
(0.001 \%) \text { mixed at a } \\
\text { ratio of } 9: 1 \\
\text { - Ultrasound at the belly } \\
\text { of the biceps brachii } \\
\text { muscle, } \\
-1 \mathrm{MHz}, 1.0 \mathrm{~W} / \mathrm{cm} 2 \text {, } \\
2.5 \mathrm{~cm} / \mathrm{s}, 10 \mathrm{~min}\end{array}$ & $\begin{array}{l}\text { (B) } \\
\text { - Ultrasound in } \\
\text { the same way as } \\
\text { group A, } \\
\text { with pure } \\
\text { ultrasound gel } \\
\text { without BV }\end{array}$ & $\begin{array}{l}\text { (1) VAS } \\
\text { (2) CK } \\
\text { (3) ROM-flexion, } \\
\text { extension }\end{array}$ & $\begin{array}{l}\text { (1) } p<0.05 \\
\text { (2) NS } \\
\text { (3) } p<0.05\end{array}$ \\
\hline $\begin{array}{l}\text { Polycystic } \\
\text { ovary } \\
\text { syndrome }\end{array}$ & $\begin{array}{l}\text { Yasin et al. } \\
\text { [26] } 2019\end{array}$ & Egypt & $\begin{array}{l}\text { (A) } 26.0 \\
\text { (B) } 26.3\end{array}$ & $\begin{array}{l}\text { (A) } 0 / 23 \\
\text { (B) } 0 / 23\end{array}$ & 14 weeks & $\begin{array}{l}\text { Ultrasound } \\
\text { gel }\end{array}$ & $\begin{array}{l}\text { (A) } \\
\text { - BV gel topical } \\
\text { application with } \\
\text { phonophoresis at BL23 } \\
\text { and Zigong } \\
\text { - } 0.6-1.0 \mathrm{mg} \text { of BV each } \\
\text { session, } 1 \mathrm{MHz} \\
\text { - twice a week } \\
\text { - low calorie diet } \\
\text { (1200-1400 kcal/day) }\end{array}$ & $\begin{array}{l}\text { (B) } \\
\text { - Ultrasound in } \\
\text { the same way as } \\
\text { group A, with } \\
\text { pure ultrasound } \\
\text { gel without BV } \\
\text { - low calorie diet } \\
\text { (1200-1400 } \\
\text { kcal/day) }\end{array}$ & $\begin{array}{l}\text { (1) } \mathrm{LH} \\
\text { (2) FSH } \\
\text { (3) LH/FSH } \\
\text { (4) Progesterone }\end{array}$ & $\begin{array}{l}\text { (1) } p=0.683 \\
\text { (2) } p=0.449 \\
\text { (3) } p=0.456 \\
\text { (4) } p=0.183\end{array}$ \\
\hline
\end{tabular}

BV: Bee Venom; BVA: Bee Venom Acupuncture; UPDRS: Unified Parkinson's Disease Rating Scale; PDQL: Parkinson's Disease Quality of Life Questionnaire; BDI: Beck Depression Inventory; BBS: Berg Balance Scale; ADL: Activities of Daily Living ; BREF: Batterie Rapide D'évaluation Frontale ; MMS: Mini Mental State; PDQ-39: Parkinson's Disease Questionnaire-39; PIGD: Postural Instability and Gait Disorder; MXE: Maximum excursion ; DCL: Directional control VAS: Visual Analog Scale; ODQ: Oswestry Disability Questionnaire; ODI: Oswestry Disability Index; EQ-5D: EuroQol 5-Dimension ; SPADI: Shoulder Pain and Disability Index; ROM: Range Of Motion; CK: Creatine Kinase; WOMAC: Western Ontario and McMaster Universities Arthritis Index; PGA: Patient Global Assessment; NS: Not Significant; NR: Not Reported. 


\subsection{Risk of Bias of Included Studies}

The quality of the included studies was assessed by the RoB tool, which consists of seven areas. Seven RCTs [16-18,22-25] were blinded by using placebo BV, accordingly, the performance bias of those seven studies were assessed as 'low'. The performance bias of the Yasin et al. study [26] was determined as 'unclear' because that study was single-blinded. Since a random sequence with even or odd numbers was generated in the Mohamed et al. study [20], the risk of selection bias of that study was determined to be 'high'. There were no descriptions about blinding in the follow-up study; therefore, the risk of performance bias and risk of detection bias for Park et al. study [19] were assessed to be 'unclear'. The risk of attrition bias of the Conrad et al. study [21] was determined to be 'high' because of the high drop-out rate. Additionally, a risk of other bias, such as that related to improper funding sources, did not exist. The RoB details are presented in Figure 2.

(B)

(A)
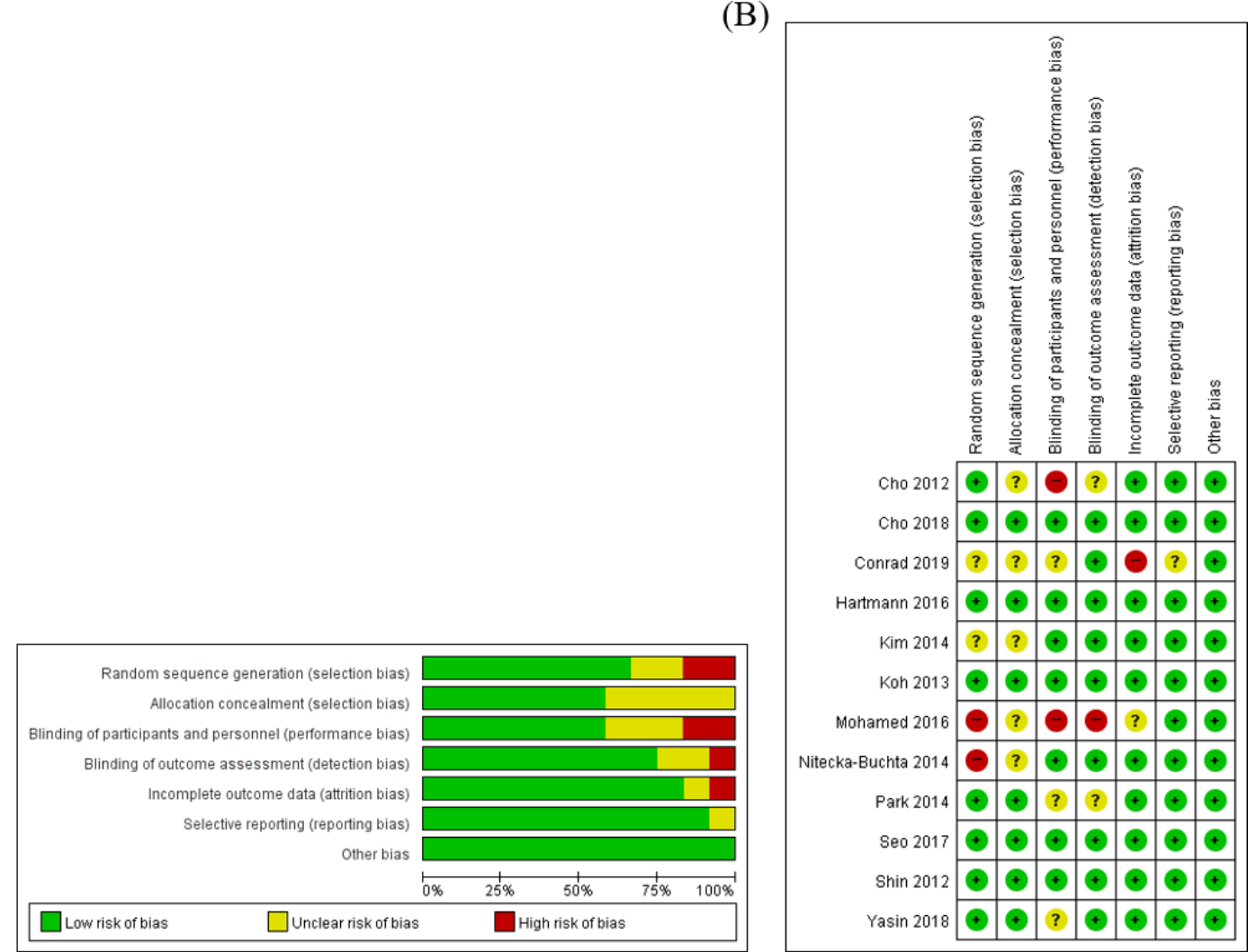

Figure 2. Methodological quality graph. (A) Risk of bias graph: authors' assessments about each risk of bias item presented as percentages of all included studies. (B): Risk of bias summary: authors' assessments about each risk of bias item for each included study. " +".

\subsection{Effectiveness of the Interventions}

\subsubsection{Parkinson's Disease}

Among the twelve RCTs included, three RCTs [15-17] evaluated the effectiveness of BVA for the treatment of Parkinson's disease. The BVA group showed a significant improvement in the UPDRS score in two studies, while BVA was not more effective than the placebo in the Hartmanss et al. study [15-17]. A qualitative synthesis could not be conducted because different outcome measures were used, and the Cho et al. (2012) [15] study reported median values only and did not report mean values. 


\subsubsection{Arthritis}

Three RCTs on adhesive capsulitis [18], pelvic inflammatory disease [20], and knee osteoarthritis [21] were included, and there was a follow-up study [19] on adhesive capsulitis. The patients with adhesive capsulitis showed improvement in the SPADI score after 12 weeks of treatment and at the 1-year follow-up. The C-reactive protein level and intensity of pain in women with pelvic inflammatory diseases significantly decreased after BV gel was topically applied with an ultrasound device and doxycycline was administered. The WOMAC score in the patients with knee arthritis also improved significantly after bee venom injections.

\subsubsection{Musculoskeletal Disorder}

There were two trials on low back pain [22,23], one trial [24] on temporomandibular disorder (RDC/TMD Ia and RDC/TMD Ib), and one trial [25] on delayed-onset muscle soreness. BVA decreased the intensity of low back pain but did not significantly increase quality of life in low back pain patients. $\mathrm{BV}$ ultrasound gel has been shown to be effective in relieving pain and improving range of motion (ROM) in patients with delayed-onset muscle soreness. Although the Nitecka-Buchta et al. study [24] did not report the $p$ value for the comparison between the two groups, BV ointment was more effective than Vaseline in improving muscle tonus and contractions in patients with temporomandibular disorder.

\subsubsection{Polycystic Ovary Syndrome}

One study [26] evaluated the hormonal changes induced by BV phonophoresis on the low back (BL23) and abdomen (Zigong) combined with a low caloric diet in obese women with polycystic ovary syndrome. The LH/FSH ratio significantly decreased and progesterone level significantly increased after 7 weeks of phonophoresis with topical BV application.

\subsection{Adverse Events}

Adverse events were reported in six RCTs $[15,16,18,21-23]$, and there were no safety issues in one study [17]. The remaining five studies did not mention adverse events. The most common symptoms caused by bee venom were skin reactions at the injection sites, including pruritus, rash, and swelling. Systemic symptoms such as headache, nasopharyngitis, and pain in an extremity were also reported. Regarding the meta-analysis results, only itchiness occurred significantly more often in the BV group than in the control group (risk ratio $=6.68,95 \%$ confidence intervals: $2.37,18.84, p<0.0003, \mathrm{I}^{2}=19 \%$ ). Detailed information on the adverse events is shown in Table 2 and Figure 3. 
Table 2. Reported adverse events of included studies.

\begin{tabular}{|c|c|c|c|c|}
\hline \multirow{2}{*}{ Author [Ref] Year } & \multirow{2}{*}{ Condition } & \multirow{2}{*}{ Type of Bee Venom } & \multicolumn{2}{|c|}{ Adverse Events [Number of Patients] } \\
\hline & & & Bee Venom Group & Control Group \\
\hline Cho et al. [15] 2012 & Parkinson's disease & Acupuncture & itchiness 1 (drop-out) & - \\
\hline Hartmann et al. [16] 2016 & Parkinson's disease & Injection & $\begin{array}{c}\text { redness/itchiness } 165 \text { cases, } \\
\text { insomnia } 1 \text { case, nausea } 3 \text { cases, } \\
\text { fatigue } 2 \text { cases, dyskinesia } 1 \text { case } \\
\text { BV specific IgE } 18 \text { patients }(90 \%) \\
\text { BV specific IgG4 } 12 \text { patients }(60 \%)\end{array}$ & $\begin{array}{c}\text { redness/itchiness } 6 \text { cases, } \\
\text { insomnia } 1 \text {, nausea } 9 \text { cases, } \\
\text { fatigue } 10 \text { cases, } \\
\text { dyskinesia } 1 \text { case, } \\
\text { bradycardia } 2 \text { cases }\end{array}$ \\
\hline Shin et al. [22] 2012 & Low back pain & Acupuncture & $\begin{array}{l}\text { itchiness } 15 \text {, skin flare 5, } \\
\text { edema } 4 \text {, rash } 2\end{array}$ & $\begin{array}{l}\text { rash } 1 \text {, headache } 1 \text {, } \\
\text { hand-foot tingling } 1\end{array}$ \\
\hline Seo et al. [23] 2017 & Low back pain & Acupuncture & $\begin{array}{c}\text { minimal itching sensation (recovered } \\
\text { completely without any treatment) } 4 \text {, } \\
\text { headache } 1, \\
\text { generalized myalgia } 1\end{array}$ & $\begin{array}{l}\text { headache } 2, \\
\text { dizziness } 2\end{array}$ \\
\hline Koh et al. [18] 2013 & Adhesive capsulitis & Acupuncture & $\begin{array}{c}\text { Muller Grade } 0 \text { reactions 30, } \\
\text { Muller Grade } 1 \text { reactions } 1\end{array}$ & slight redness and pruritus 3 \\
\hline Conrad et al. [21] 2019 & Knee osteoarthritis & Injection & $\begin{array}{c}\text { swelling } 15 \text {, discoloration } 10, \text { pruritus } 8, \\
\text { erythema } 7, \text { urticaria } 5, \\
\text { hypersensitivity } 3, \text { edema } 3, \\
\text { injection site pain } 3 \text {, vesicles } 3, \\
\text { rash } 1, \text { hematoma } 1 \text {, paresthesia } 1, \\
\text { headache } 24, \text { back pain } 13, \\
\text { nasopharyngitis } 11, \\
\text { upper respiratory tract infection } 11, \text { pain in } \\
\text { extremity } 10, \text { arthralgia } 8, \text { diarrhea } 10\end{array}$ & $\begin{array}{l}\text { itchiness } 2, \text { erythema } 1, \\
\text { urticaria } 1 \text {, rash } 1, \\
\text { headache } 17, \text { back pain } 9, \\
\text { nasopharyngitis } 7, \\
\text { upper respiratory tract infection } 4, \\
\text { pain in extremity } 4, \\
\text { arthralgia } 4 \text {, diarrhea } 1\end{array}$ \\
\hline
\end{tabular}




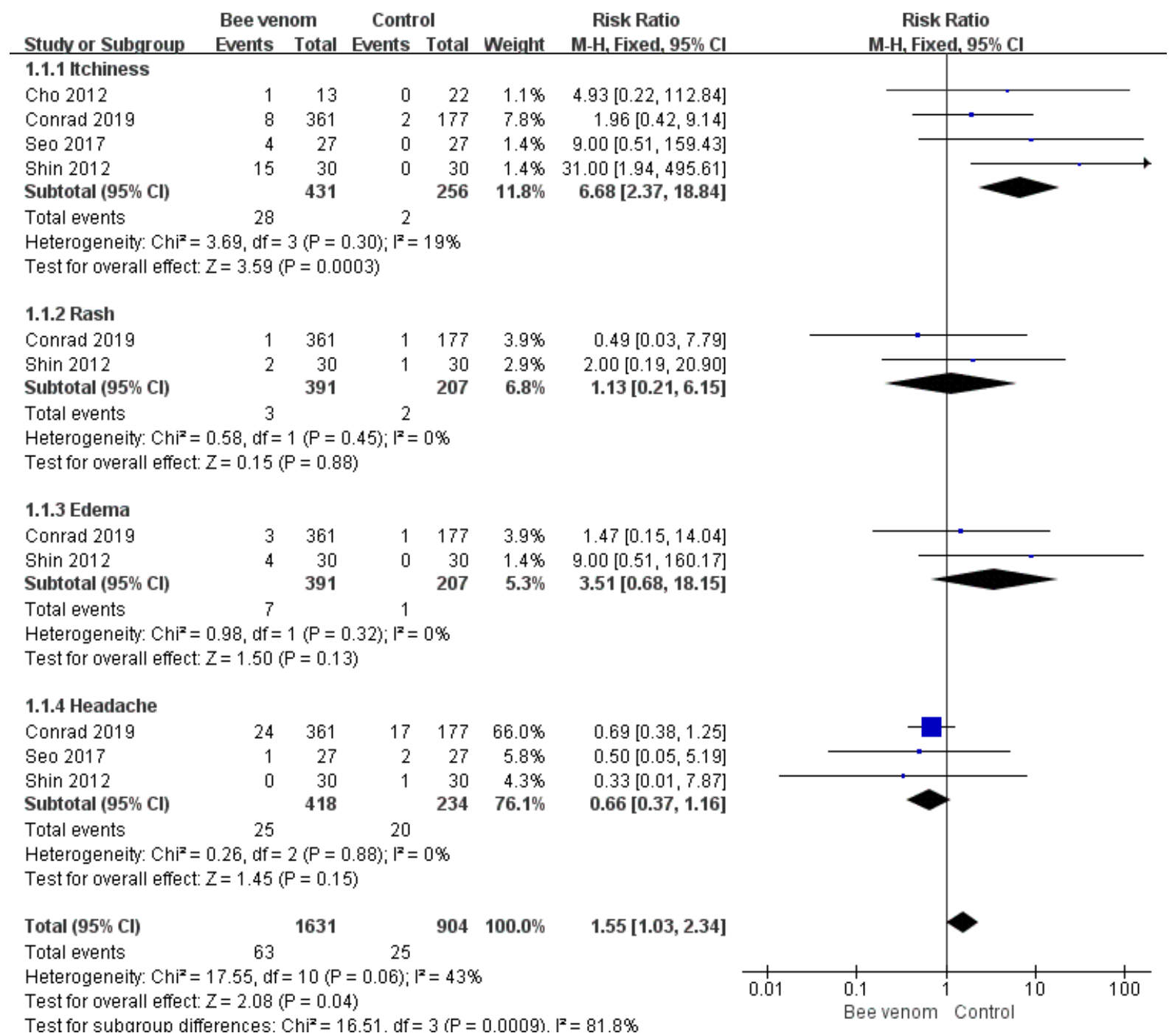

Figure 3. Risk ratio of reported adverse events of bee venom therapy.

\section{Discussion}

BVT is a method of treating diseases that utilizes the pharmacological actions of bee venom, which is used by bees for self-defense, and is widely used in countries worldwide, including China, Korea, Germany, the United Kingdom, Switzerland and France [27]. BVT involving the injection of bee venom at acupoints is called BVA; WHO defined this type of BVT as "a special type of acupuncture performed by bee stings at a certain point or cutaneous region of the meridian/channel for therapeutic purposes, particularly for pain relief" [28]. The venom used for BVA is extracted from the poisons of Apis Mellifera using a bee venom collector.

In this systematic review, twelve RCTs were included, and they were divided into three categories on the basis of the diseases treated-Parkinson's disease, arthritis, and musculoskeletal disorders. Among the three RCTs on Parkinson's disease, the treatment frequency for the two studies [15,17] showing improvement in the UPDRS score was twice a week. On the other hand, the remaining study [16] showed no effects induced by BVT performed once a month. The frequency of treatment for Parkinson's disease is recommended to be 2-3 times a week; accordingly, if BVA was administered more frequently in Hartmanss et al.'s study, the results may have been more favorable. The former two studies selected acupuncture points (GB20, LI11, GB34, ST36); however, the latter did not report the injection site. Although there are several preclinical studies $[29,30]$ that have shown the effects of BV on central nervous system diseases such as Parkinson's disease and multiple sclerosis, the mechanism 
of BV has not yet been clearly revealed. BVA is assumed to inhibit the development or progression of dysfunction of the central nervous system, such as those observed in patients with PD, multiple sclerosis, and amyotrophic physical sclerosis [30]. However, since the mechanism remains unclear, it is necessary to conduct research on the mechanism as well as on the clinical effects of BVA.

Two studies [18,19] assessed the effects of BVA on adhesive capsulitis; one was a 12-week RCT [18], and the other [19] was a 1-year follow-up RCT. The SPADI scorer significantly improved after 12 weeks of treatment as well as at the one-year follow-up. BVA was also performed for knee osteoarthritis in one RCT, and it was confirmed that various WOMAC scores improved. One RCT reported that BV gel phonophoresis for pelvic inflammation significantly reduced the C-reactive protein level and reduced the pain intensity. BVA, in nonclinical studies, can improve rheumatoid arthritis [31]. Melittin, which is one component of bee venom, has been attracting as an alternative treatment for rheumatoid arthritis due to its anti-inflammatory effects [32]. It also improved degenerative osteoarthritis of the knee joint [33], arthritis of the elbow joint [34], and hip joint disease in case reports and clinical reviews [35].

Generally, arachidonic acid stimulates the production of cyclooxygenase during intracellular metabolic processes and promotes the synthesis of prostaglandins to induce inflammatory reactions [36]. Then, prostaglandin E2 stimulates bradykinin and histamine in afferent nerve terminal receptors, causing sensory hypertrophy with pain and inflammatory reactions in joints, such as edema and vascular dilation [37]. It has been reported that BV inhibits COX-2 and prostaglandin E2 in the body, suggesting that it may be effective in treating inflammation in various parts of a joint [33]. In particular, the treatment effects have been reported in dogs with hip osteoarthritis [38], indicating that this treatment can be applied not only to humans but also to animals.

The efficacy of BVA in treating low back pain was studied in two RCTs [22,23], both of which showed that BVA significantly reduces pain intensity. There was one RCT [25] that revealed the effect of ultrasound gel with diluted BV on delayed-onset muscle soreness, and the VAS pain score and ROM improved. There was one RCT [24] that showed no significant effects of BV ointment on temporomandibular disorder. BVA involves BV being injected directly into the skin, but BV gel phonophoresis and BV ointment are indirect methods. However, during phonophoresis, transdermal drug delivery is enhanced by ultrasound waves [39]. For ointments, it is difficult for the large-molecular-weight BV particles to penetrate the skin, which may have limited their effects. Therefore, although BV injections may be effective because they are highly absorbent, they cause severe pain [40], and BV phonophoresis [25] is suggested as an alternative. A comparative study of the effects of BV injections and BV phonophoresis should be conducted to determine whether they are safe and effective treatment methods. In particular, the fact that Korean medicine doctors use many pharmacopuncture treatments for musculoskeletal diseases (frequency of $41.7 \%$ ), neurological diseases $(18.0 \%)$, and gastrointestinal diseases $(9 \%)$ indirectly shows that BVA affects musculoskeletal disorders [41].

A total of six RCTs $[15,16,18,21-23]$ reported adverse events, which were mostly minor and transient skin reactions, such as pruritus, rash, and swelling. Well-known severe side effects such as anaphylaxis may have been obscured in RCTs with relatively small sample sizes, due to a low incidence of $0.014 \%$ [42]. It is also thought that patients with a high risk of hypersensitivity or anaphylaxis were excluded after skin tests and patient histories were performed. In addition, in some studies, granulomas or plaques were observed weeks or months after the BVA treatment [3,43], but in RCTs, the follow-up period is short, so it is less likely to observe these adverse events within the study period.

There are several limitations in this study. First, only those written in English were included in this review. The studies written Chinse or Korean had been omitted. Second, eight of the twelve RCTs included in the study were carried out in Korea, and it is difficult to have sufficient external validity, so this issue should be considered when the results are interpreted or generalized to other populations. Third, it was not discussed that the roles of bee venom compounds in connection to included diseases. This review focused on summarizing the published randomized control trials on the clinical effectiveness of BVT. Fourth, studies targeting cancer patients were excluded although 
there are references that bee venom has anticancer effects [32]. Since many cancer patients generally get chemotherapy, the authors of this study were concerned that the adverse events of bee venom therapy might be exaggerated. Fourth the number of the included RCTs was not large, and adverse reactions that have a low probability of occurring or are delayed may not have been observed and therefore reported within the short follow-up period.

\section{Conclusions}

This study reviewed the clinical effectiveness of and AEs induced by bee venom treatment. Despite causing mild skin reactions such as pruritus, rash, and swelling, bee venom showed therapeutic effectiveness in treating inflammatory arthritis and musculoskeletal diseases. This study suggests that large-scale clinical trials on bee venom need to be conducted and that a reporting system for AEs needs to be developed to enhance the validity of bee venom treatment.

Author Contributions: Conceptualization, K.H.K., and S.J.; methodology, S.J.; software, S.J.; validation, S.J., and K.H.K.; formal analysis, S.J.; investigation, S.J., and K.H.K.; resources, K.H.K.; data curation, K.H.K.; writing-original draft preparation, S.J.; writing-review and editing, K.H.K.; visualization, S.J.; supervision, K.H.K.; project administration, K.H.K.; funding acquisition, K.H.K. All authors have read and agreed to the published version of the manuscript.

Funding: This work was funded by National Research Foundation of Korea (NRF-2019R1C1C1007573).

Conflicts of Interest: The authors declare that there are no conflicts of interest regarding the publication of this paper.

Availability of Data and Materials: The data used for this study are available from the corresponding author upon request.

\section{References}

1. Son, D.J.; Lee, J.W.; Lee, Y.H.; Song, H.S.; Lee, C.K.; Hong, J.T. Therapeutic application of anti-arthritis, pain-releasing, and anti-cancer effects of bee venom and its constituent compounds. Pharmacol. Ther. 2007, 115, 246-270. [CrossRef]

2. Korean Pharmacopuncutre Institute. Pharmacopuncturology, 3rd ed.; Hanmi Medical Publishing Co.: Seoul, Korea, 2019.

3. Park, J.S.; Lee, M.J.; Chung, K.H.; Ko, D.K.; Chung, H. Live bee acupuncture (Bong-Chim) dermatitis: Dermatitis due to live bee acupuncture therapy in Korea. Int. J. Dermatol. 2013, 52, 1519-1524. [CrossRef] [PubMed]

4. Chen, J.; Lariviere, W.R. The nociceptive and anti-nociceptive effects of bee venom injection and therapy: A double-edged sword. Prog. Neurobiol. 2010, 92, 151-183. [CrossRef] [PubMed]

5. Lee, G.; Bae, H. Bee Venom Phospholipase A2: Yesterday's Enemy Becomes Today's Friend. Toxins 2016, 8, 48. [CrossRef]

6. Zhang, S.; Liu, Y.; Ye, Y.; Wang, X.-R.; Lin, L.-T.; Xiao, L.-Y.; Zhou, P.; Shi, G.-X.; Liu, C.-Z. Bee venom therapy: Potential mechanisms and therapeutic applications. Toxicon 2018, 148, 64-73. [CrossRef] [PubMed]

7. Lee, M.S.; Pittler, M.H.; Shin, B.C.; Kong, J.C.; Ernst, E. Bee venom acupuncture for musculoskeletal pain: A review. J. Pain. 2008, 9, 289-297. [CrossRef] [PubMed]

8. Choi, M.S.; Park, S.; Choi, T.; Lee, G.; Haam, K.K.; Hong, M.C.; Min, B.I.; Bae, H. Bee venom ameliorates ovalbumin induced allergic asthma via modulating CD4+CD25+ regulatory T cells in mice. Cytokine 2013, 61, 256-265. [CrossRef] [PubMed]

9. Lee, J.Y.; Kang, S.S.; Kim, J.-H.; Bae, C.S.; Choi, S.H. Inhibitory effect of whole bee venom in adjuvant-induced arthritis. Vivo 2005, 19, 801-805.

10. Baek, Y.H.; Huh, J.E.; Lee, J.D.; Choi, D.Y.; Park, D.S. Antinociceptive effect and the mechanism of bee venom acupuncture (Apipuncture) on inflammatory pain in the rat model of collagen-induced arthritis: Mediation by alpha2-Adrenoceptors. Brain Res. 2006, 1073-1074, 305-310. [CrossRef]

11. Chen,H.-S.; Qu, F.; He, X.; Liao, D.; Kang, S.M.; Lu, S.J. The anti-nociceptive effect and the possible mechanism of acupoint stimulation caused by chemical irritants in the bee venom pain model. Brain Res. 2010, 1355, 61-69. [CrossRef] 
12. Park, J.H.; Yim, B.K.; Lee, J.-H.; Lee, S.; Kim, T.-H. Risk associated with bee venom therapy: A systematic review and meta-analysis. PLoS ONE 2015, 10, e0126971. [CrossRef] [PubMed]

13. Shamseer, L.; Moher, D.; Clarke, M.; Ghersi, D.; Liberati, A.; Petticrew, M.; Shekelle, P.; Stewart, L.A.; PRISMA-P Group. Preferred reporting items for systematic review and meta-analysis protocols (PRISMA-P) 2015: Elaboration and explanation. BMJ 2015, 2015, g7647. [CrossRef] [PubMed]

14. Cumpston, M.; Li, T.; Page, M.J.; Chandler, J.; Welch, V.A.; Higgins, J.P.; Thomas, J. Updated guidance for trusted systematic reviews: A new edition of the Cochrane Handbook for Systematic Reviews of Interventions. Cochrane Database Syst. Rev. 2019, 10, ED000142. [CrossRef]

15. Cho, S.-Y.; Shim, S.-R.; Rhee, H.Y.; Park, H.-J.; Jung, W.-S.; Moon, S.-K.; Park, J.-M.; Ko, C.-N.; Cho, K.-H.; Park, S.-U. Effectiveness of acupuncture and bee venom acupuncture in idiopathic Parkinson's disease. Parkinsonism. Relat. Disord. 2012, 18, 948-952. [CrossRef]

16. Hartmann, A.; Mullner, J.; Meier, N.; Hesekamp, H.; van Meerbeeck, P.; Habert, M.-O.; Kas, A.; Tanguy, M.-L.; Mazmanian, M.; Oya, H.; et al. Bee Venom for the Treatment of Parkinson Disease-A Randomized Controlled Clinical Trial. PLoS ONE 2016, 11, e0158235. [CrossRef]

17. Cho, S.-Y.; Lee, Y.-E.; Doo, K.-H.; Lee, J.-H.; Jung, W.-S.; Moon, S.-K.; Park, J.-M.; Ko, C.-N.; Kim, H.; Rhee, H.Y.; et al. Efficacy of Combined Treatment with Acupuncture and Bee Venom Acupuncture as an Adjunctive Treatment for Parkinson's Disease. J. Altern. Complem. Med. 2018, 24, 25-32. [CrossRef] [PubMed]

18. Koh, P.S.; Seo, B.K.; Cho, N.S.; Park, H.S.; Park, D.S.; Baek, Y.H. Clinical effectiveness of bee venom acupuncture and physiotherapy in the treatment of adhesive capsulitis: A randomized controlled trial. J. Shoulder. Elb. Surg. 2013, 22, 1053-1062. [CrossRef]

19. Park, Y.C.; Koh, P.S.; Seo, B.K.; Lee, J.W.; Cho, N.S.; Park, H.S.; Park, D.S.; Baek, Y.H. Long-term effectiveness of bee venom acupuncture and physiotherapy in the treatment of adhesive capsulitis: A one-year follow-up analysis of a previous randomized controlled trial. J. Altern. Complement. Med. 2014, 20, 919-924. [CrossRef]

20. Mohamed, E.A.; Ewida, M.M. Efficacy of bee venom phonphoresis in treatment of chronic pelvic inflammatory diseases. Int. J. PharmTech Res. 2016, 9, 66-71.

21. Conrad, V.J.; Hazan, L.L.; Latorre, A.J.; Jakubowska, A.; Kim, C.M.H. Efficacy and Safety of Honey Bee Venom (Apis mellifera) Dermal Injections to Treat Osteoarthritis Knee Pain and Physical Disability: A Randomized Controlled Trial. J. Altern. Complement. Med. 2019, 25, 845-855. [CrossRef]

22. Shin, B.-C.; Kong, J.C.; Park, T.-Y.; Yang, C.-Y.; Kang, K.-W.; Choi, S.-M. Bee venom acupuncture for chronic low back pain: A randomised, sham-controlled, triple-blind clinical trial. Eur. J. Integr. Med. 2012, 4, E271-E280. [CrossRef]

23. Seo, B.-K.; Han, K.; Kwon, O.; Jo, D.-J.; Lee, J.-H. Efficacy of Bee Venom Acupuncture for Chronic Low Back Pain: A Randomized, Double-Blinded, Sham-Controlled Trial. Toxins 2017, 9, 361. [CrossRef] [PubMed]

24. Nitecka-Buchta, A.; Buchta, P.; Tabenska-Bosakowska, E.; Walczynska-Dragon, K.; Baron, S. Myorelaxant effect of bee venom topical skin application in patients with RDC/TMD Ia and RDC/TMD Ib: A randomized, double blinded study. Biomed. Res. Int. 2014, 2014, 296053. [CrossRef]

25. Kim, S.K.; Kim, M.C. The affect on delayed onset muscle soreness recovery for ultrasound with bee venom. J. Phys. Ther. Sci. 2014, 26, 1419-1421. [CrossRef] [PubMed]

26. Yasin, M.M.; Elhosary, E.A.; Hamada, H.A.; Yousef, A.M.; Shahin, M.; Mosaad, D. Effect of bee venom phonophoresis in obese polycystic ovarian women: A Single Blind Randomized Controlled Trial. J. Appl. Pharm. Sci. 2018, 8, 159-164. [CrossRef]

27. Ahn, C.B.; Youn, H.M.; Cho, E.J. An Investigation of Directions of Research on Bee Venom in the Sphere of Oriental Medicine in Korea in Last Decade. J. Dong-Eui Orient. Med. 2001, 5, 23-42.

28. World Health Organization Regional Office for the Western Pacific (WPRO). WHO International Standard Terminologies on Traditional Medicine in the Western Pacific Region; WPRO: Manila, Philippines, 2007; Available online: https://apps.who.int/iris/bitstream/handle/10665/206952/9789290612487_eng.pdf? sequence $=1 \&$ is Allowed $=y$ (accessed on 20 June 2020).

29. Castro, H.J.; Mendez-Inocencio, J.I.; Omidvar, B.; Omidvar, J.; Santilli, J.; Nielsen, H.S.; Pavot, A.P.; Richert, J.R.; Bellanti, J.A. A phase I study of the safety of honeybee venom extract as a possible treatment for patients with progressive forms of multiple sclerosis. Allergy. Asthma Proc. 2005, 26, 470-476.

30. Park, W.; Kim, J.K.; Kim, J.-I.; Choi, D.-Y.; Koh, H.-K. Neuroprotective and Anti-inflammatory Effects of Bee Venom Acupuncture on MPTP-induced Mouse. J. Acupunct. Res. 2010, 27, 105-116. 
31. You, D.-S.; Kwon, Y.-D. The Effect of Bee Venom Pharmacopuncture Therapy on the Condition of Different Concentration in Rheumatoid Arthritis Rat Model. J. Orient. Rehab. Med. 2011, 21, 101-123. [CrossRef]

32. Aufschnaiter, A.; Kohler, V.; Khalifa, S.; El-Wahed, A.A.; Du, M.; El-Seedi, H.; Buttner, S. Apitoxin and Its Components against Cancer, Neurodegeneration and Rheumatoid Arthritis: Limitations and Possibilities. Toxins 2020, 12, 66. [CrossRef]

33. Lim, J.A.; Kim, S.C.; Kim, S.N.; Lee, S.Y.; Moon, H.C.; Shin, M.S.; Kim, H.J.; Koo, S.T.; Choi, S.M. The Clinical Study on Bee Venom Acupuncture Treatment on Osteoarthritis of Knee Joint. J. Pharmacopunct. 2005, 20, 73-81. [CrossRef]

34. Heo, D.S.; Keum, D.H. Effect of Intra-articular Bee Venom Injection on Acute Traumatic Arthritis of Elbow Joint: Two Cases Report. J. Orient. Rehab. Med. 2005, 15, 171-180.

35. Kim, T.-H.; Kang, K.-S.; Kwon, G.-R. Three cases of affections of the hip treated with Korean Bee-Venom therapy. J. Pharmacopunct. 2001, 4, 127-134. [CrossRef]

36. Surh, Y.-J.; Chun, K.-S.; Cha, H.-H.; Han, S.S.; Keum, Y.-S.; Park, K.-K.; Lee, S.S. Molecular mechanisms underlying chemopreventive activities of anti-inflammatory phytochemicals: Down-regulation of COX-2 and iNOS through suppression of NF-kappa B activation. Mutat. Res. 2001, 480-481, 243-268. [CrossRef]

37. Chen, S.-H.; Fahmi, H.; Shi, Q.; Benderdour, M. Regulation of microsomal prostaglandin E2 synthase-1 and 5-lipoxygenase-activating protein/5-lipoxygenase by 4-hydroxynonenal in human osteoarthritic chondrocytes. Arthritis Res. Ther. 2010, 12, R21. [CrossRef] [PubMed]

38. Kim, T.-H.; Kim, B.-Y.; Kim, W.-B.; Kim, K.-S.; Liu, J.; Kim, D.-H. Bee-venom acupuncture treatment of hip osteoarthritis in a dog. J. Vet. Clin. 2006, 23, 190-193.

39. Cagnie, B.; Vinck, E.; Rimbaut, S.; Vanderstraeten, G. Phonophoresis versus topical application of ketoprofen: Comparison between tissue and plasma levels. Phys. Ther. 2003, 83, 707-712. [CrossRef]

40. Kwon, Y.B.; Lee, H.J.; Han, H.J.; Mar, W.C.; Kang, S.K.; Yoon, O.B.; Beitz, A.J.; Lee, J.H. The water-soluble fraction of bee venom produces antinociceptive and anti-inflammatory effects on rheumatoid arthritis in rats. Life Sci. 2002, 71, 191-204. [CrossRef]

41. Park, J.E.; Kim, K.-H.; Kang, S.; Lee, E.K.; Kim, J.-C.; Jang, B.-H.; Shin, Y.-C.; Ko, S.-G. Usage status and satisfaction with pharmacopuncture in Korea: A survey among Korean medicine doctors. Eur. J. Integr. Med. 2019, 27, 121-130. [CrossRef]

42. Yang, M.-S.; Lee, S.-H.; Kim, T.-W.; Kwon, J.-W.; Lee, S.-M.; Kim, S.-H.; Kwon, H.-S.; Park, C.-H.; Park, H.-W.; Kim, S.-S.; et al. Epidemiologic and clinical features of anaphylaxis in Korea. Ann. Allerg. Asthma. Im. 2008, 100, 31-36. [CrossRef]

43. Shim, W.-H.; Park, H.-J.; Kim, H.-S.; Chin, H.-W.; Kim, S.-H.; Ko, H.-C.; Kim, B.-S.; Kim, M.-B.; Kwon, K.-S. Mybacterium chelonae Infection Occurring at the Site of Bee Sting Therapy. Korean J. Dermatol. 2011, 49, 374-378.

(C) 2020 by the authors. Licensee MDPI, Basel, Switzerland. This article is an open access article distributed under the terms and conditions of the Creative Commons Attribution (CC BY) license (http://creativecommons.org/licenses/by/4.0/). 